\section{Bugscope: The Second Year Of A Sustainable Remote Microscope Project For K-12 Educational Outreach}

C.S. Potter, B. Carragher, L. Carroll, C. Conway*, B. Grosser, J. Hanlon, N. Kisseberth, S. Robinson, D. Stone*,

U. Thakkar** and D. Weber.

Beckman Institute, *University High School and ${ }^{\star \star}$ National

Center for Supercomputing Applications, University of Illinois

Bugscope ${ }^{1}$ is a second generation educational project in the World Wide Laboratory ${ }^{2}$ that provides web browser based control of scientific imaging instrumentation using the Internet. We had previously demonstrated web based remote access to sophisticated scientific imaging systems several years ago in the Chickscope project ${ }^{3,4}$. The primary goal of the Bugscope project is to demonstrate that relatively low cost, sustainable access to an environmental scanning electron microscope (ESEM) can be made available to K-12 classrooms.

Methods: To participate in the project, a classroom submits a web based application that describes how they plan to use the microscope. If the application is accepted, a one hour session on the ESEM is scheduled and the classroom mails in their chosen specimen. During their access time, classrooms use a standard web browser over the Internet to control and acquire images from the ESEM (Philips/FEI XL-30FEG). The interface is shown in Figure 1 and the classroom can control stage movement, magnification $(10-10,000 x)$, focus and contrast. Images, including any annotations and comments made by the participants, are automatically stored to a database along with the associated acquisition parameters. The database is immediately accessible from the Bugscope web site to any interested observer. The irma software allows for several classrooms to simultaneously and independently access and control the microscope thus providing the potential for supporting multiple classrooms during each session. The effectiveness of the Bugscope project is evaluated by requesting background information from the classroom teacher prior to the project, feedback immediately following the session, and a project report a week after the session.

To achieve the goal of low cost, sustainable access to the instrument, it is clear that professional university staff can not be involved in the weekly operational aspects of the project. In the Bugscope project this problem has been addressed by training a team of high school students and undergraduates to prepare the specimens and perform the initial microscope setup. Another key aspect of sustaining the project has been to automate many of the required project administration and data handling tasks. An infrastructure has been developed so that applications submitted by the teachers through the Web are automatically archived into a database. These are then reviewed and scheduled, using a Web based interface, by the project team and the results progress of the experiment. are automatically collected to provide an overall merit value for the proposed project. This information is then used to set up and schedule a session for the classrooms. During the session the acquired images are also automatically stored and can be later retrieved by the classroom (or any other interested group) though $\vec{a}$ the Web. Without this automation, sustaining this project in the long term would be overwhelming and would require the assistance of a full time project administrator.

Results and Conclusions: As of February 2000, we have supported over 30 classrooms (approximately 1000 students) from all over the country. We have had enthusiastic response from the students and teachers that have participated in the project and $\frac{\bar{\sigma}}{\bar{\sigma}}$ over 100 applications have been submitted. We currently routinely $\frac{7}{0}$ support two classrooms a week and we expect to support approxi- $\frac{\circ}{\rho}$ mately 100 sessions a year. The project requires minimal staff resources and less than 3 hours of instrument time a week.

The most obvious benefit of the Bugscope project is that is provides students with access to scientific instrumentation and expertise that would not otherwise be available to them. More importantly the project engages students in the scientific process and gives them some experience of the realities of conducting scientific research. In planning the experiment and providing the speci- $\frac{0}{0}$ mens, students have a sense of ownership of the project that ${ }^{4}$ would be very difficult to duplicate using a simulator or a virtual machine. This benefit was pointed out numerous times in the final evaluations submitted by the participating teachers.

Addendum: As of $9 / 20 / 0044$ schools have participated in the project.

\section{1. http://bugscope.beckman.uiuc.edu}

2. Carragher, B. and Potter, C.S. (1999). The World Wide Laboratory: Remote and Automated Access to Imaging Instrumentation. In Impact of Advances in Computing and Communications in Chemical Sciences and Technology, pp.141-153, National Academy Press, Washington, DC

3. http://chickscope. beckman.uiuc.edu

4. Bruce, B.C., B.O. Carragher, B.M. Damon, M.J. Dawson, J.A. Eurell, C. D. Gregory, P.C. Lauterbur, M.M. Marjanovic, B. Mason-Fossum, H.D. Morris, C.S. Potter, U. Thakkar, (1997). Chickscope: An Interactive MRI Classroom Curriculum Innovation for $\mathrm{K}-12$, Computers and Education Journal $29,73-87$
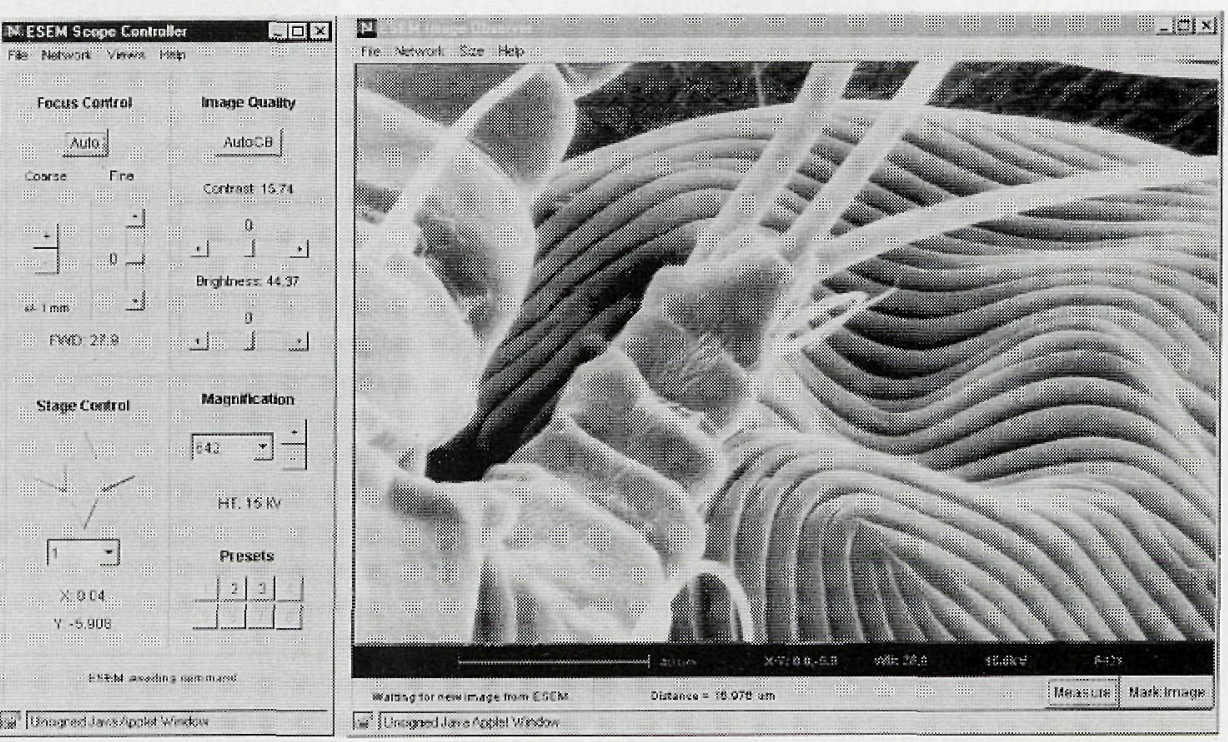

Figure 1: The IRMA Microscope Controller (left) and Image Observer (right). These Java Applets are launched by a standard web browser from a classroom computer and used to interface the students to the instrument via the internet. The Observer may also be launched by any group interested in watching the

Microsc. Microanal. 6 (Suppl 2: Proceedings): 1160-1161 (2000); reprinted with the permission of the publisher, Springer-Verlag, NY. 


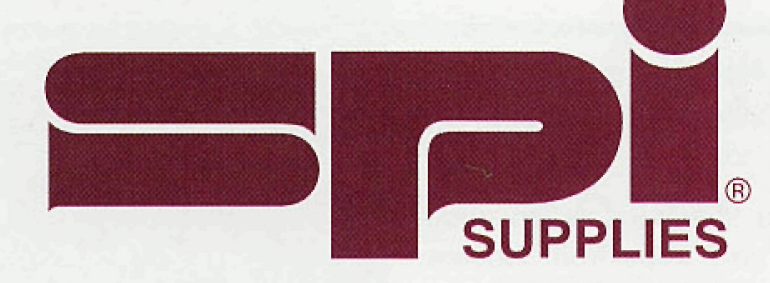

File Edit Uiew Go Bookmarks Options Directory Help

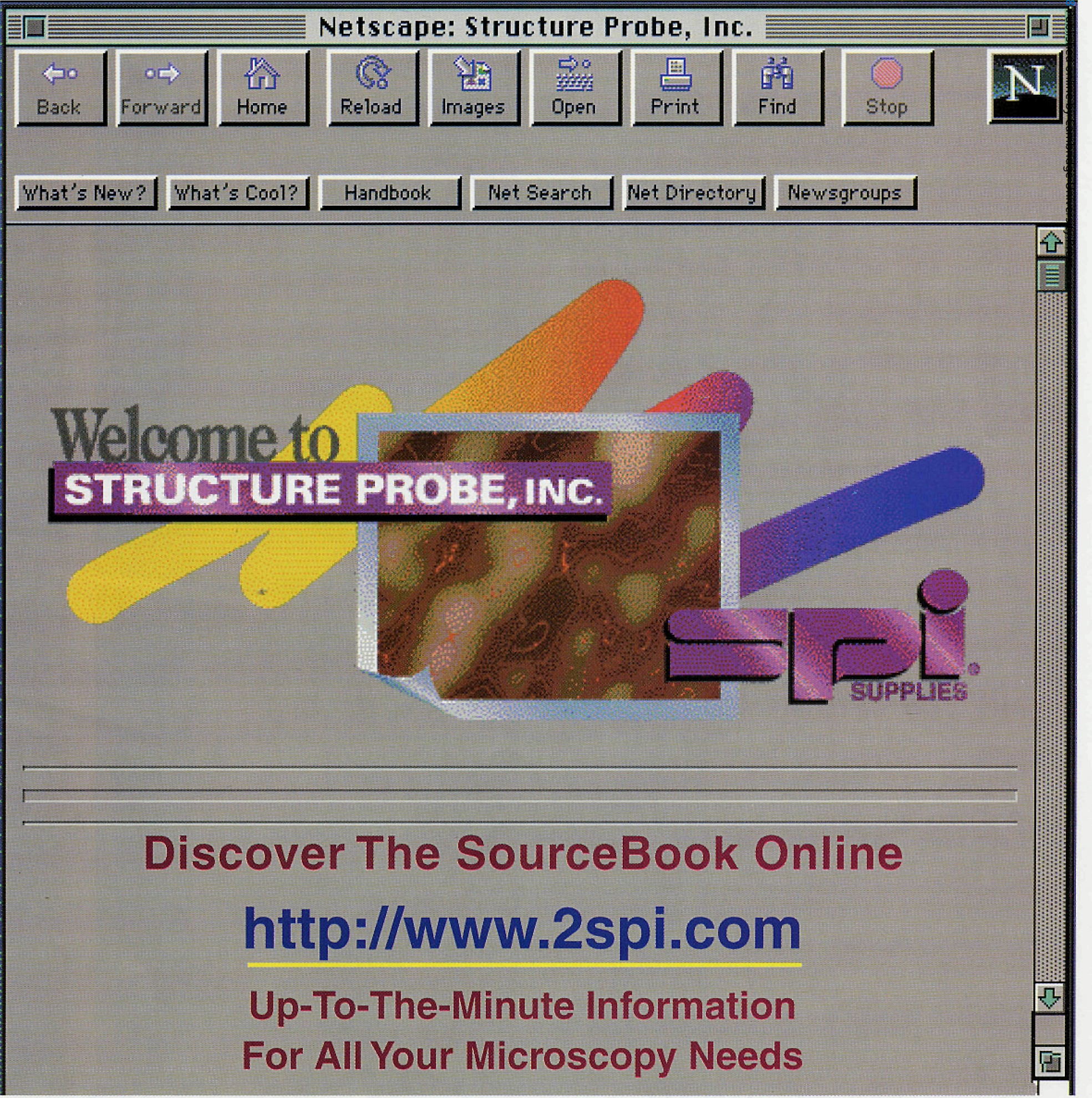

SPI Supplies Division of STRUCTURE PROBE, Inc.

P.O. Box 656 - West Chester, PA 19381-0656 USA

Ph.: 1-610-436-5400 • 1-800-2424-SPI (U.S. only) • FAX: 1-610-436-5755 • E-mail: spi2spi@ 2spi.com 